\title{
Determination of the Relative Energies of Activation for the Dissociation of Aromatic Versus Aliphatic Phosphopeptides by ESI-FTICR-MS and IRMPD
}

\author{
Jason W. Flora \\ Deparatment of Chemistry, Virginia Commonwealth University, Richmond, Virginia, USA
}

\section{David C. Muddiman}

W. M. Keck FT-ICR Mass Spectrometry Laboratory, Mayo Proteomics Research Center and the Department of Biochemistry and Molecular Biology, Mayo Clinic College of Medicine, Rochester, Minnesota, USA

Fourier Transform Ion Cyclotron Resonance Mass Spectrometry (ESI-FTICR-MS) coupled with infrared multiphoton dissociation (IRMPD) is potentially a powerful method for rapid phosphopeptide mapping of complex proteolytic digests. The dissociation of deprotonated phosphopeptides by IRMPD is energetically favorable over unmodified deprotonated peptides because of a lower energy of activation and a higher internal energy under identical irradiation conditions. The energies of activation for dissociation are determined for model peptides phosphorylated on an aliphatic side chain (serine) and an aromatic side chain (tyrosine). The determination of phosphorylation location provides important biochemical information identifying the kinase involved in specific phosphorylation mechanisms. The data presented in this manuscript also support the theory that for phosphopeptides, the phosphate moiety's P-O stretch is in direct resonance with the infrared laser $(10.6 \mu \mathrm{m})$, thus increasing the relative absorptivity of the modified species. A greater extinction coefficient affords more extensive photon absorption and subsequently a greater internal energy at the rapid exchange limit. (J Am Soc Mass Spectrom 2004, 15, 121-127) (c) 2004 American Society for Mass Spectrometry

$\mathrm{P}$ hosphorylation is a reversible posttranslational modification that predominantly controls protein activity in eucaryotic cells. The phosphorylation reaction is catalyzed by kinases and involves the exothermic transfer of a phosphate group $(80 \mathrm{Da})$ from adenosine triphosphate (ATP) to the hydroxyl group of the aliphatic or aromatic side chains of serine/threonine or tyrosine, respectively. This modification is governed by two classes of protein kinases, serine/threonine $(\mathrm{S} / \mathrm{T})$ kinases and tyrosine $(\mathrm{Y})$ kinases. In physiological conditions, the phosphate group contains two negative charges promoting significant conformational changes to the protein's overall structure. These structural changes ultimately regulate the activity of the kinase receptor proteins. This covalent protein modification is responsible for the regulation of cellular processes such as metabolism, growth, and reproduction [1-3] and an ever-increasing number of diseases have been associated with mutation or miss expression of protein ki-

Published online December 2, 2003

Address reprint requests to Dr D. C. Muddiman, Department of Biochemistry and Molecular Biology, Medical Sciences Building 3-115, Mayo Clinic College of Medicine, Rochester, MN 55905, USA. E-mail: muddiman.david@mayo.edu nases [2]. Subsequently, accurate determination of the site and level of phosphorylation of target proteins provides valuable insight regarding many biological regulatory mechanisms and diseases as well as what kinases are involved in the cell signaling process [4,5].

As mass spectrometry's role in proteomics has expanded exponentially, it has become an increasingly important approach for the accurate determination of the site of phosphorylation. Several novel techniques have arisen to address the identification of this essential posttranslational modification. However, this regulatory modification presents a formidable challenge to the mass spectrometrist because it is usually present in low abundances in biological mixtures, and the modified peptides are difficult to ionize with respect to unmodified peptides. Because of the challenges presented by phosphopeptide detection, several methods have been derived in the interest of phosphopeptide enrichment from proteolytic digests. Fractionation techniques prior to mass analysis include reverse phase HPLC [6], immobilized metal ion affinity chromatography (IMAC) $[7,8]$, chemically tagging the phosphate with an affinity tag (i.e., biotin) [9-12], and immunoprecipitation [1316]. Several methods of phosphopeptide mapping have 
also been proposed, taking advantage of the relative speed, sensitivity, and adaptability of MS. These methods include the use of phosphatases [17-20], ${ }^{31} \mathrm{P}$ monitoring by inductively couple plasma (ICP)-MS [21], collision induced dissociation (CID) [22-25], precursor ion scanning [24, 26-31], neutral loss scanning [32], nozzle-skimmer dissociation [33, 34], metastable decomposition [35-37], and electron capture [38].

A method for phosphopeptide mapping of complex proteolytic digests using ESI [39] FTICR-MS [40] and IRMPD [41] has recently been developed in this laboratory $[42,43]$. This approach provides a rapid, selective, and sensitive means of phosphopeptide detection where initial research has demonstrated that within a complex tryptic digest, phosphopeptides can be simultaneously identified by a single IR laser irradiation event [42]. Based upon the energetics of dissociation, ESI-FTICR-MS with IRMPD of deprotonated proteolytic digests in a Penning ion trap may be an ideal platform for phosphopeptide mapping [42, 43]. IRMPD preferentially dissociates deprotonated phosphopeptides over deprotonated unmodified peptides of identical amino acid sequence [43]. The irradiation power required to dissociate the phosphopeptides is significantly less than unmodified analogs due to the lower energy of activation $\left(E_{a}\right)$ for dissociation of the posttranslationally modified species.

A recent study [43] suggests that the $E_{a}$ for dissociation is not the only factor contributing to the selective dissociation of the phosphopeptides by IRMPD. The $\mathrm{P}-\mathrm{O}$ stretch $\left(9.6\right.$ to $11 \mu \mathrm{m}$ or 1042 to $909 \mathrm{~cm}^{-1}$ ) of this important modification is in direct resonance with the $\mathrm{CO}_{2}$ IR laser (10.57 to $10.63 \mu \mathrm{m}$ or 940.7 to $946.1 \mathrm{~cm}^{-1}$ ) used for IRMPD. Subsequently, more frequent absorption of photons under identical irradiation conditions can result for the modified peptide over the unmodified analog, affording a higher internal energy at the rapid exchange limit (REX) [43-45]. This theory is supported by an early account of IRMPD for biomolecular sequencing by McLafferty and coworkers [41], where they reported that the structure of an ion is very important for the time required to reach the "onset of fragmentation". It was stated that oligonucleotides best demonstrated this fact [41]. The rapid dissociation of oligonucleotides by IRMPD is proposed to be attributable to the low $\mathrm{E}_{\mathrm{a}}$ for dissociation of the phosphodiester backbone "and/or" the direct resonance of the IR laser photons to the $\mathrm{P}-\mathrm{O}$ stretch of the phosphodiester backbone.

Marshall and coworkers [45] previously determined that IRMPD of ions with vibrational frequencies close to the laser irradiation frequency absorb energy more readily than ions with more distant overall vibrational frequencies. The peptides used for Marshall's experiments did not; however, contain a strong absorbing functional group for the wavenumber $943 \mathrm{~cm}^{-1}$. Therefore, when one considers two analogous amino acid sequences where one contains one or more functionalities that strongly absorb IR photons at the same irradiation frequency of the laser used for IRMPD, more frequent absorption of photons under that identical irradiation conditions would result.

The $E_{a}$ for dissociation of phosphopeptides is the physical reason for the selective dissociation of phosphopeptides; however, the vibrational frequency of the phosphate moiety can enable more efficient photon absorption than the unmodified peptide under identical irradiation conditions. The resulting higher internal energy of the phosphopeptide achieved during IR irradiation, due to the strong absorbing $\mathrm{P}-\mathrm{O}$ stretch in resonance with the IR laser, contribute to a more rapid first-order decay of the excited precursor ion relative to unmodified peptides.

Previous CID studies have determined that phosphorylated serines and threonines dissociate through $\beta$-elimination, losing $\mathrm{H}_{3} \mathrm{PO}_{4}$ and forming dehydroalanine and dehydroaminobutyric acid, respectively [25]. Phosphotyrosines, in contrast, cannot undergo the same $\beta$-elimination because of their aromatic side chain and subsequently, CID of phosphotyrosine peptides results in the loss of $\mathrm{H}_{3} \mathrm{PO}_{4}, \mathrm{H}_{2} \mathrm{O}$, and $\mathrm{HPO}_{3}$. However, it has been observed that for some amino acid sequences, particularly at high charge states, peptides phosphorylated in tyrosines may experience only backbone cleavage with no loss of the phosphate moiety [25]. Furthermore, it has been observed that phosphothreonine peptides can lose $\mathrm{HPO}_{3}$ through direct dephosphorylation as a competing dissociation channel to the $\beta$-elimination mechanism [25]. Therefore, for dissociation of unknown peptides, the loss of $98 \mathrm{Da}$ gives rise to signature ions of phosphopeptides; however, the lack of phosphate loss (98 Da) cannot confirm that a phosphopeptide is not present. Additionally, signature ions cannot be used with any certainty to identify the phosphorylated amino acid. Therefore, it is important to determine if the energetics of dissociation can be used to delineate between phosphorylation of an aliphatic or aromatic side chain, providing information about the kinase involved in the regulatory mechanism under investigation.

\section{Experimental}

The aromatic and aliphatic 7-mer phosphopeptides, GAG-pY-GAG (pY) and GAG-pS-GAG (pS), were custom synthesized by New England Peptide, Inc. (Fitchburg, MA). Methanol and piperidine were purchased from Sigma-Aldrich (St. Louis, MO). Water was purified to $18 \mathrm{M} \Omega$ with a Barnstead Nanopure Infinity (Dubuque, Iowa) ultrapure water system.

All experiments were conducted on a modified Ionspec (Irvine, CA) ESI-FTICR-MS with a 7 tesla magnetic field using previously described microspray sources [46]. ESI infusion was performed using a Harvard syringe pump model PHD 2000 with ESI solutions consisting of $50 \%$ methanol, $50 \%$ water, and a final concentration of $20 \mathrm{mM}$ piperidine. Infusion was carried out at a rate of $3.3 \mathrm{~nL} / \mathrm{s}$ through a $20 \mu \mathrm{L}$ Hamilton syringe held at a potential of $-1.5 \mathrm{keV}$. All acquisitions 
were made in the negative-ion mode and all data involved single hexapole accumulations (ranging from 2 to $4 \mathrm{~s}$ ) followed by gated trapping [47].

Following ESI, hexapole accumulation, and gated trapping in the ICR cell, IRMPD was conducted on the trapped ions with a 25 watt Synrad 48-2(W) (Mukilteo, WA) $\mathrm{CO}_{2}$ laser. The laser wavelength was $10.6 \mu \mathrm{m}(2.83$ $\times 10^{13} \mathrm{~Hz}$ ) with a beam diameter of $3.5 \mathrm{~mm}$. This laser is fitted with a $2.5 \times$ diffuser, which was directed through a $\mathrm{BaF}_{2}$ window into the ICR cell. For all experiments, the entire ion population trapped in the ICR cell was uniformly irradiated as the diffused laser beam has a diameter of $8.75 \mathrm{~mm}$. Assuming ambient temperatures prior to irradiation at a 7 tesla magnetic field, the thermal radius of the ion cloud was $\sim 0.08 \mathrm{~mm}$ for the singly-charged 7-mers (pY and pS). Following an induction period, the dissociation data obtained from IRMPD can be applied to first-order kinetics (vide infra). The induction period can be defined as the time required to increase the precursor ions' internal energy from that analogous of ambient temperatures (298 K) to an energy sufficient to promote the lowest energy of activation dissociation pathway [44]. At the laser powers used for the experiments discussed herein, the dissociation rates are slow enough that the ions reach an internal "temperature" where a Boltzmann distribution of internal energy is achieved and the ions are at the radiative exchange (REX) limit [44].

All Fourier transform infrared spectroscopy (FTIR) experiments were conducted using a Nicolet Nexus 670 (Thermo Nicolet Corporation, Madison, WI) with a multibounce attenuated total reflectance (ATR) module with 20 $\mu \mathrm{L}$ of $1 \mathrm{mM}$ 50:50 methanol:water solutions dried on the ATR plate (150 spectra).

\section{Results and Discussion}

The application of mass spectrometry for the unimolecular dissociation of trapped gas-phase ions provides valuable information about the ion's dissociation energetics. Dunbar [48] first determined bond dissociation energies using IR irradiation produced by continuous wave (CW) $\mathrm{CO}_{2}$ laser for small molecules. Dunbar and McMahon [49] later demonstrated that a vacuum chamber (ion trap) can act as a blackbody source of IR photons when heated to a particular temperature. Marshall and coworkers recently demonstrated that IRMPD can be used to determine the relative $\mathrm{E}_{\mathrm{a}}$ for dissociation of large gas-phase biomolecules (>50 atoms) analogous to values previously determined by BIRD [45]. Hannis and Muddiman [50] have applied this technique to the determination of the relative $\mathrm{E}_{\mathrm{a}}$ for dissociation of oligonucleotides and modified (7-deaza purine) oligonucleotides.

Relative energies of activation $\left(E_{a}\right)$ for gas-phase dissociation of GAG-pS-GAG and GAG-pY-GAG are determined by ESI-FTICR-MS with IRMPD of trapped ions and the equation derived by Dunbar [51] based upon the approximate relation between laser intensity and temperature where $v$ is the frequency of radiation $\left(10.6 \mu \mathrm{m}=2.83 \times 10^{13} \mathrm{~Hz}\right), h$ is Plank's constants, $q$ is the partition function (1.05) [45], and $I_{\text {laser }}$ is the laser intensity $\left(\mathrm{Wcm}^{-2}\right)$ :

$$
E_{a}=-\frac{d \ln k_{\mathrm{diss}}}{d 1 /(k T)}=q h v \frac{d \ln k_{\mathrm{diss}}}{d \ln I_{\text {laser }}}
$$

First-order rate constants $\left(k_{\text {diss }}\right)$ were obtained from the slopes of the natural log of the precursor relative ion abundance versus irradiation time for five laser powers (W). Eq 1 can be rearranged where $E_{a}$ can be determined from a plot the natural $\log$ of $\mathrm{k}_{\text {diss }}$ versus the natural $\log$ of laser intensity $\left(\mathrm{W} \mathrm{cm}^{-2}\right)$ :

$$
\ln k_{\mathrm{diss}}=\frac{E_{a}}{q h v} \ln I_{\text {laser }}
$$

Because neighboring amino acid functional groups could affect the relative $E_{a}$, this possibility must be eliminated for model systems when evaluating the difference between modified aliphatic and aromatic amino acid dissociation. The two model systems from which the relative $E_{a}$ for dissociation will be determined are GAG-pS-GAG and GAG-pY-GAG. This peptide is analogous to the size of a small tryptic fragment; however they lack a Lys or Arg C-terminus to significantly reduce the potential for unique side chain interactions with the dissociation mechanisms. Because of the low molecular weights of these model systems, 555 and 631, respectively, only singly-charged species were detected, providing further simplicity of the system.

Figure 1 shows the first-order rate constant plots of the natural $\log$ of the relative ion abundance versus the irradiation time for both peptides. The laser irradiation powers for GAG-pS-GAG peptide are 2, 2.5, 3, 3.5, and 4 watts while GAG-pY-GAG involved 1.5, 2, 2.5, 3, and 3.5 watts. Previous studies have revealed that peptides phosphorylated on the tyrosines tend to be more stable than those phosphorylated on serines and threonines because of a lack of $\beta$-elimination pathways during collisional activation [52]. Curiously, this did not seem to be the case during the IRMPD experiments involving GAGT-pS-GAG and GAG-pY-GAG. The tyrosine phosphopeptide dissociated significantly faster rate than the serine phosphopeptide under identical irradiation conditions. However, based upon these $\mathrm{E}_{\mathrm{a}}$ for dissociation calculation, Figure 2, these modified 7-mers have relatively the same energy of activation, $0.48 \mathrm{eV}$ and 0.50 $\mathrm{eV}$, respectively.

Figure 3a shows a plot of the natural log of the relative ion abundance versus the irradiation time for both peptides at 2 watts. Clearly, the tyrosine phosphopeptide dissociates at a faster rate than the serine phosphopeptide where $50 \%$ of GAG-pS-GAG is dissociated after $4.5 \mathrm{~s}$ of irradiation at 2 watts and GAG-pYGAG only after $1.5 \mathrm{~s}$. Figure $3 \mathrm{~b}$ shows the ESI-FTICR mass spectrum of GAG-pY-GAG after $1 \mathrm{~s}$ of IRMPD at 


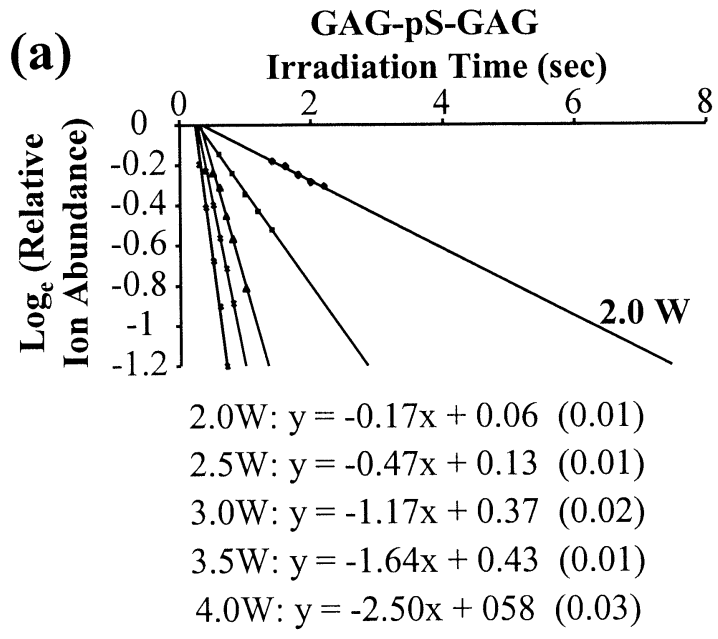

(b)

GAG-pY-GAG

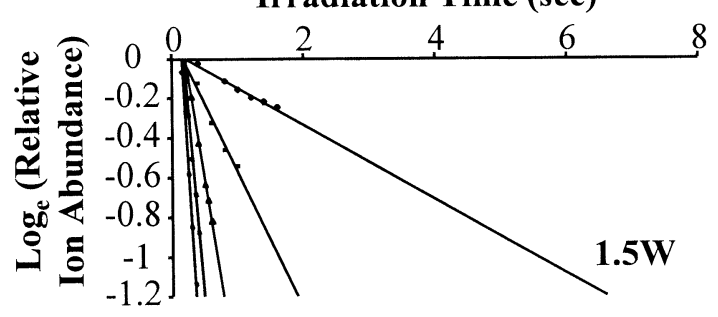

$$
\begin{aligned}
& 1.5 W: y=-0.07 x+0.12(0.01) \\
& 2.0 W: y=-0.19 x+0.04(0.03) \\
& 2.5 W: y=-2.07 x+0.42(0.02) \\
& \text { 3.0W: } y=-3.87 x+0.67(0.01) \\
& 3.5 W: y=-5.42 x+0.78(0.03)
\end{aligned}
$$

Figure 1. First-order rate constants are obtained from the slopes of the natural $\log$ of the relative ion abundance versus the irradiation time (seconds) for: (a) the GAG-pS-GAG at laser irradiation powers of 2 (diamond), 2.5 (square), 3 (triangle), 3.5 (x), and 4 (star) watts and (b) the GAG-pY-GAG at laser irradiation powers of 1.5 (diamond), 2.0 (square), 2.5 (triangle), $3.0(\mathrm{x})$, and 3.5 (star) watts. Each line equation is listed with its irradiation power (watts) and the standard error of a predicted $\mathrm{y}$ for a given $\mathrm{x}$ in the regression is given in parenthesis.

2 watts where $58 \%$ of the precursor ion relative ion intensity remains. Figure 3c shows the mass spectrum of the GAG-pS-GAG where $90 \%$ of the precursor ion of this phosphopeptide remains after IRMPD for $1 \mathrm{~s}$ at 2 watts. Under identical isolation events and irradiation conditions, GAG-pY-GAG is clearly dissociating more rapidly. To confirm that these observations were not an artifact of the isolation event, the phosphopeptides were electrosprayed simultaneously and irradiated without ion isolation. The same phenomenon (more significant GAG-pY-GAG dissociation) was observed (data not shown), and this is in direct accord with the observations noted above.

Figure 4 is the normalized attenuated total reflectance FTIR spectra of the two modified 7-mers and identifies the potential reason for the difference in
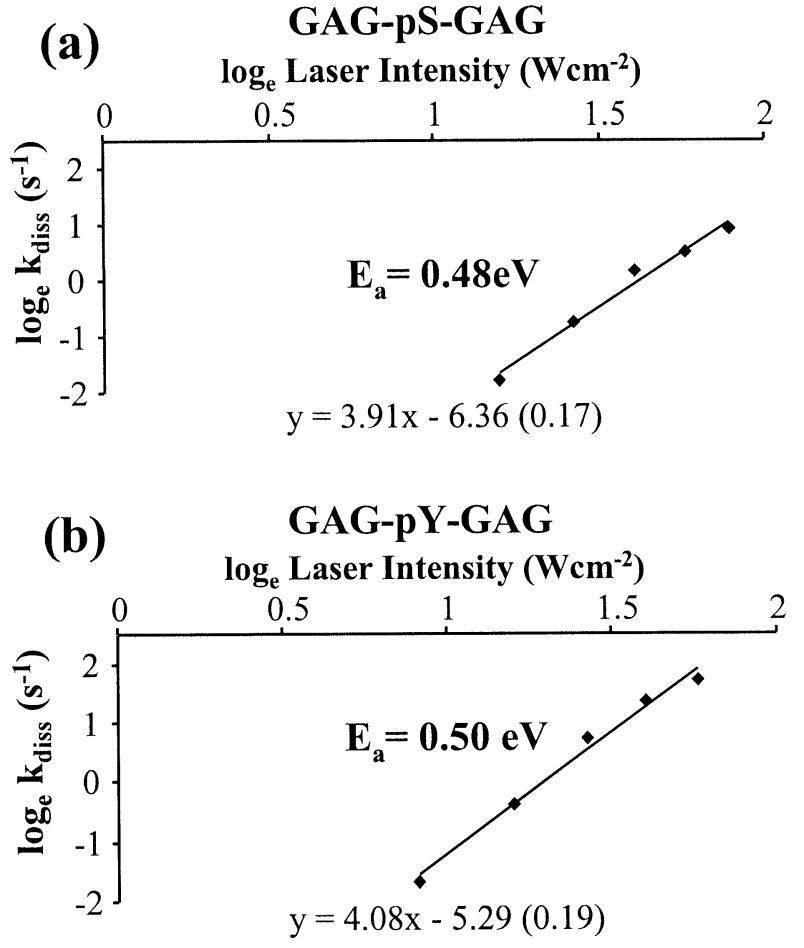

Figure 2. The energies of activation obtained from the slope of the natural log of the rate constants plotted against the natural log of the laser intensity $\left(\mathrm{Wcm}^{-2}\right)$. The relative energy of activation for dissociation are shown for (a) the GAG-pS-GAG and (b) the GAG-pY-GAG .

dissociation rate under identical irradiation parameters. The relative absorptivity for the phosphorylation of the aromatic side chain (pY) is greater than the aliphatic side chain (pS). The extinction coefficients $(k)$, determined from the absorbance mode assuming reflectance is proportional to the transmittance and $100 \%$ of the reflectance corresponds to $100 \%$ of the transmittance, of pY relative to $\mathrm{pS}(k=1)$ at the frequency of the IR irradiation is 1.3 . While this $k$ value represents only a moderate increase in absorptivity, it significantly contributes to the rate of first-order decay of the precursor ion population of the phosphopeptide modified on the tyrosine. Therefore, under identical irradiation parameters the peptide phosphorylated on the tyrosine has a higher internal energy than the peptide phosphorylated on the serine due to more efficient photon absorption at the frequency of irradiation, accounting for a faster dissociation rate even with equivalent energies of activation.

\section{Conclusions}

ESI-FTICR-MS with IRMPD is an ideal platform for phosphopeptide detection because the $\mathrm{E}_{\mathrm{a}}$ for the dissociation of phosphopeptides is lower than that of the unmodified analogs. Furthermore, the vibrational mode of the phosphate moiety greatly increases absorbance and, as a result, the kinetics of dissociation for these 


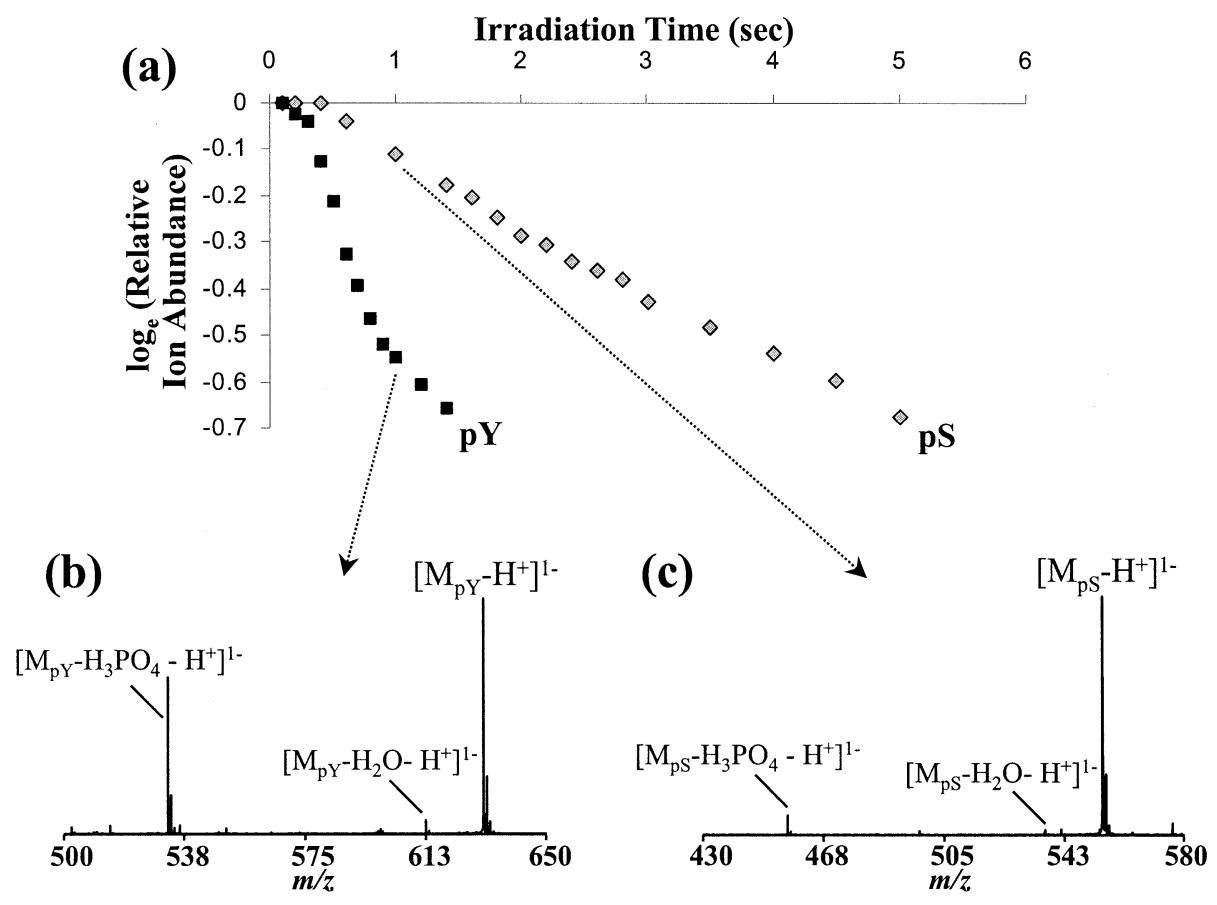

Figure 3. (a) Plot of the natural log of the relative ion abundance versus the irradiation time (seconds) for GAG-pS-GAG (pS) (diamonds) and GAG-pY-GAG (pY) (squares) peptides at 2 watts. (b) GAG-pY-GAG product ion spectra after $1 \mathrm{~s}$ of irradiation at 2 watts. (c) GAG-pS-GAG product ion spectra after $1 \mathrm{~s}$ of irradiation at 2 watts.

modified peptides. This theory is supported by the data presented in this manuscript. While GAG-pY-GAG and GAG-pS-GAG have analogous $\mathrm{E}_{\mathrm{a}}$ for dissocation, the aromatic phosphonate dissociates at a significantly greater rate of dissociation than the aliphatic species under identical irradiation conditions which is attributed to its absorptivity of the IR photons is higher.

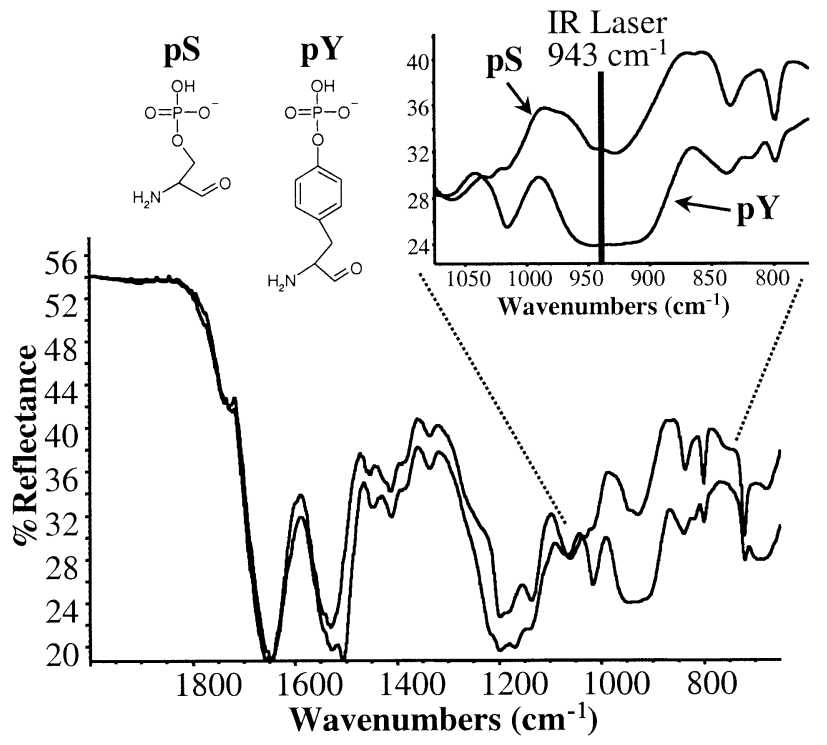

Figure 4. Normalized ATR-FTIR spectra of GAG-pS-GAG (pS) (black line) and GAG-pY-GAG (pY) (gray line). The inset shows the region of the $\mathrm{P}-\mathrm{O}$ stretch enlarged and the wavenumber (943 $\mathrm{cm}^{-1}$ ) of the laser used for IRMPD.
Ideally, computational determination (e.g., master equation modeling of the overall vibrational frequency and steady-state Boltzman temperature at a fixed laser intensity) would provide valuable support to these conclusions. These data suggest that it is possible to distinguish a peptide containing a single phosphorylation event residing on a serine/threonine versus a tyrosine, and vice-versa, within a complex proteolytic digest derived from a biological system.

\section{References}

1. Krebs, E. G. The Growth of Research on Protein Phosphorylation. Trends Biochem. Sci. 1994, 19, 439.

2. Hunter, T. Protein Kinases and Phosphatases: The Yin and Yang of Protein Phosphorylation and Signaling. Cell 1995, 80, 225-236.

3. Posada, J.; Cooper, J. A. Molecular Signal Integration. Interplay between Serine, Threonine, and Tyrosine Phosphorylation. Mol. Biol. Cell 1992, 3, 583-592.

4. Kennelly, P. J.; Krebs, E. G. Consensus Sequences as Substrate Specificity Determinants for Protein Kinases and Protein Phosphatases. J. Biol. Chem. 1991, 266, 15555-15558.

5. Songyang, Z.; Cantley, L. C. Recognition and Specificity in Protein Tyrosine Kinase-Mediated Signalling. Trends Biochem. Sci. 1995, 20, 470-475.

6. Davis, M. T.; Lee, T. D. Rapid Protein Identification Using a Microscale Electrospray LC/MS System on an Ion Trap Mass Spectrometer. J. Am. Soc. Mass Spectrom. 1998, 9, 194-201.

7. Zhou, W.; Merrick, B. A.; Khaledi, M. G.; Tomer, K. B. Detection and Sequencing of Phosphopeptides Affinity Bound to Immobilized Metal Ion Beads by Matrix-Assisted Laser Desorption/Ionization Mass Spectrometry. J. Am. Soc. Mass Spectrom 1999, 11, 273-282. 
8. Posewitz, M. C.; Tempst, P. Immobilized Gallium(III) Affinity Chromatography of Phosphopeptides. Anal. Chem. 1999, 71, 2883-2892.

9. McLachlin, D. T.; Chait, B. T. Analysis of Phosphorylated Proteins and Peptides by Mass Spectrometry. Curr. Opin. Chem. Biol. 2001, 5, 591-602.

10. Oda, Y.; Nagasu, T.; Chait, B. T. Enrichment Analysis of Phosphorylated Proteins as a Tool for Probing the Phosphoproteome. Nat. Biotechnol. 2001, 19, 379-382.

11. Zhou, H.; Watts, J. D.; Aebersold, R. A Systematic Approach to the Analysis of Protein Phosphorylation. Nat. Biotechnol. 2001, 19, 375-378.

12. Goshe, M. B.; Conrads, T. P.; Panisko, E. A.; Angell, N. H.; Veenstra, T. D.; Smith, R. D. Phosphoprotein Isotope-Coded Affinity Tag Approach for Isolating and Quantitating Phosphopeptides in Proteome-Wide Analyses. Anal. Chem. 2001, 73, 2578-2586.

13. Kalo, M. S.; Pasquale, E. B. Multiple in Vivo Tyrosine Phosphorylation Sites in Ephb Receptors. Biochemistry 1999, 38, 14396-14408.

14. Watty, A.; Neubauer, G.; Dreger, M.; Zimmer, M.; Wilm, M.; Burden, S. J. The in Vitro and in Vivo Phosphotyrosine Map of Activated Musk. Proc. Natl. Acad. Sci. U.S.A. 2000, 97, 45854590.

15. Okamura, H.; Aramburu, J.; Garcia-Rodriguez, C.; Viola, J. P.; Raghavan, A.; Tahiliani, M.; Zhang, X.; Qin, J.; Hogan, P. G.; Rao, A. Concerted Dephosphorylation of the Transcription Factor Nfat1 Induces a Conformational Switch that Regulates Transcriptional Activity. Mol. Cell 2000, 6, 539-550.

16. Knotts, T. A.; Orkiszewski, R. S.; Cook, R. G.; Edwards, D. P.; Weigel, N. L. Identification of a Phosphorylation Site in the Hinge Region of the Human Progesterone Receptor and Additional Amino-Terminal Phosphorylation Sites. J. Biol. Chem. 2001, 276, 8475-8483.

17. Larsen, M. R.; Sorensen, G. L.; Fey, S. J.; Larsen, P. M.; Roepstorff, P. Phospho-Proteomics: Evaluation of the Use of Enzymatic De-Phosphorylation and Differential Mass Spectrometric Peptide Mass Mapping for Site Specific Phosphorylation Assignment in Proteins Separated by Gel Electrophoresis. Proteomics 2001, 1, 223-238.

18. Liao, P. C.; Leykam, J.; Andrews, P. C.; Gage, D. A.; Allison, J. An Approach to Locate Phosphorylation Sites in a Phosphoprotein: Mass Mapping by Combining Specific Enzymatic Degradation with Matrix-Assisted Laser Desorption/Ionization Mass Spectrometry. Anal. Biochem. 1994, 219, 9-20.

19. Jensen, O. N.; Larsen, M. R.; Roepstorff, P. Mass Spectrometric Identification and Microcharacterization of Proteins from Electrophoretic Gels: Strategies and Applications. Proteins 1998(Suppl 2), 74-89.

20. Amankwa, L. N.; Harder, K.; Jirik, F.; Aebersold, R. HighSensitivity Determination of Tyrosine-Phosphorylated Peptides by on-Line Enzyme Reactor and Electrospray Ionization Mass Spectrometry. Protein Sci. 1995, 4, 113-25.

21. Wind, M.; Edler, M.; Jakubowski, N.; Linscheid, M.; Wesch, H.; Lehmann, W. D. Analysis of Protein Phosphorylation by Capillary Liquid Chromatography Coupled to Element Mass Spectrometry with 31p Detection and to Electrospray Mass Spectrometry. Anal. Chem. 2001, 73, 29-35.

22. Covey, T.; Shushan, B.; Bonner, R.; Schröder, W.; Hucho, F. LC/MS and LC/MS/MS Screening for the Sites of PostTranslational Modification in Proteins. In Methods in Protein Sequence Analysis (Proceedings of the 8th International Conference), Jörnvall, H.; Höög, J.-O.; Gustavsson, A.-M., Eds.; Birkhäuser Verlag; Basel, 1991; pp. 249-256.

23. Tholey, A.; Reed, J.; Lehmann, W. D. Electrospray Tandem Mass Spectrometric Studies of Phosphopeptides and Phosphopeptide Analogues. J. Mass Spectrom. 1999, 34, 117-123.
24. Annan, R. S.; Huddleston, M. J.; Verma, R.; Deshaies, R. J.; Carr, S. A. A Multidimensional Electrospray MS-Based Approach to Phosphopeptide Mapping. Anal. Chem. 2001, 73, 393-404.

25. DeGnore, J. P.; Qin, J. Fragmentation of Phosphopeptides in an Ion Trap Mass Spectrometer. J. Am. Soc. Mass Spectrom. 1998, 9 , 1175-1188.

26. Wilm, M.; Neubauer, G.; Mann, M. Parent Ion Scans of Unseparated Peptide Mixtures. Anal. Chem. 1996, 68, 527-33.

27. Carr, S. A.; Huddleston, M. J.; Annan, R. S. Selective Detection and Sequencing of Phosphopeptides at the Femtomole Level by Mass Spectrometry. Anal. Biochem. 1996, 239, 180-192.

28. Steen, H.; Kuster, B.; Fernandez, M.; Pandey, A.; Mann, M. Detection of Tyrosine Phosphorylated Peptides by Precursor Ion Scanning Quadrupole TOF Mass Spectrometry in Positive Ion Mode. Anal. Chem. 2001, 73, 1440-1448.

29. Borchers, C.; Parker, C. E.; Deterding, L. J.; Tomer, K. B. Preliminary Comparison of Precursor Scans and Liquid Chromatography-Tandem Mass Spectrometry on a Hybrid Quadrupole Time-of-Flight Mass Spectrometer. J. Chromatogr. A 1999, 854, 119-130.

30. Verma, R.; Annan, R. S.; Huddleston, M. J.; Carr, S. A.; Reynard, G.; Deshaies, R. J. Phosphorylation of Sic1p by G1 Cdk Required for Its Degradation and Entry into S Phase. Science 1997, 278, 455-460.

31. Beuvink, I.; Hess, D.; Flotow, H.; Hofsteenge, J.; Groner, B.; Hynes, N. E. Stat5a Serine Phosphorylation. Serine 779 is Constitutively Phosphorylated in the Mammary Gland, and Serine 725 Phosphorylation Influences Prolactin-Stimulated in Vitro DNA Binding Activity. J. Biol. Chem. 2000, 275, 1024710255.

32. Schlosser, A.; Pipkorn, R.; Bossemeyer, D.; Lehmann, W. D. Analysis of Protein Phosphorylation by a Combination of Elastase Digestion and Neutral Loss Tandem Mass Spectrometry. Anal. Chem. 2001, 73, 170-176.

33. Huddleston, M. J.; Annan, R. S.; Bean, M. F.; Carr, S. A. Selective Detection of Phosphopeptides in Complex Mixtures by Electrospray Liquid Chromatography Mass Spectrometry. J. Am. Soc. Mass Spectrom. 1993, 4, 710-717.

34. Ding, J.; Burkhart, W.; Kassel, D. B. Identification of Phosphorylated Peptides from Complex Mixtures Using Negative-Ion Orifice-Potential Stepping and Capillary Liquid Chromatography/Electrospray Ionization Mass Spectrometry. Rapid Commun. Mass Spectrom. 1994, 8, 94-98.

35. Annan, R. S.; Carr, S. A. Phosphopeptide Analysis by MatrixAssisted Laser Desorption Time-of-Flight Mass Spectrometry. Anal. Chem. 1996, 68, 3413-21.

36. Schnölzer, M.; Lehmann, W. D. Identification of Modified Peptides by Metastable Fragmentation in MALDI Mass Spectrometry. Int. J. Mass Spectrom. Ion Processes 1997, 169/170, 263-271.

37. Metzger, S.; Hoffmann, R. Studies on the Dephosphorylation of Phosphotyrosine-Containing Peptides During Post-Source Decay in Matrix-Assisted Laser Desorption/Ionization. J. Mass Spectrom. 2000, 35, 1165-1177.

38. Shi, S. D.; Hemling, M. E.; Carr, S. A.; Horn, D. M.; Lindh, I.; McLafferty, F. W. Phosphopeptide/Phosphoprotein Mapping by Electron Capture Dissociation Mass Spectrometry. Anal. Chem. 2001, 73, 19-22.

39. Fenn, J. B.; Mann, M.; Meng, C. K.; Wong, S. F.; Whitehouse, C. M. Electrospray Ionization for Mass Spectrometry of Large Biomolecules. Science 1989, 246, 64-71.

40. Comisarow, M. B.; Marshall, A. G. Fourier Transform Ion Cyclotron Resonance Mass Spectrometry. Chem. Phys. Lett. 1974, 25, 282-283.

41. Little, D. P.; Speir, J. P.; Senko, M. W.; O'Connor, P. B.; McLafferty, F. W. Infrared Multiphoton Dissociation of Large 
Multiply Charged Ions for Biomolecule Sequencing. Anal. Chem. 1994, 66, 2809-2815.

42. Flora, J. W.; Muddiman, D. C. Selective, Sensitive, and Rapid Phosphopeptide Identification in Enzymatic Digests Using ESI-FT-ICR-MS with Infrared Multiphoton Dissociation. Anal. Chem. 2001, 73, 3305-3311.

43. Flora, J. W.; Muddiman, D. C. Gas-Phase Ion Unimolecular Dissociation for Rapid Phosphopeptide Mapping by IRMPD in a Penning Ion Trap: An Energetically Favored Process. J. Am. Chem. Soc. 2002, 124, 6546-6547.

44. Jockusch, R. A.; Paech, K.; Williams, E. R. Energetics from Slow Infrared Multiphoton Dissociation of Biomolecules. J. Phys. Chem. A 2000, 104, 3188-3196.

45. Freitas, M. A.; Hendrickson, C. L.; Marshall, A. G. Determination of Relative Ordering of Activation Energies for Gas-Phase Ion Unimolecular Dissociation by Infrared Radiation for Gaseous Multiphoton Energy Transfer. J. Am. Chem. Soc. 2000, 122, 7768-7775.

46. Hannis, J. C.; Muddiman, D. C. Nanoelectrospray Mass Spectrometry Using Non-Metalized, Tapered (50-10 Mm) FusedSilica Capillaries. Rapid Commun. Mass Spectrom. 1998, 12, 443-448.
47. Senko, M. W.; Hendrickson, C. L.; Emmett, M. R.; Shi, S. D.-H.; Marshall, A. G. External Accumulation of Ions for Enhanced Electrospray Ionization Fourier Transform Ion Cyclotron Resonance Mass Spectrometry. J. Am. Soc. Mass Spectrom. 1997, 8, 970-976.

48. Dunbar, R. C. Kinetics of Thermal Unimolecular Dissociation by Ambient Infrared Radiation. J. Phys. Chem. 1994, 98, 87058712.

49. Dunbar, R. C.; McMahon, T. B. Activation of Unimolecular Reactions by Ambient Blackbody Radiation. Science 1998, 279, 194.

50. Hannis, J. C.; Muddiman, D. C. Tailoring the Gas-Phase Dissociation and Determining the Relative Energy of Activation for Dissociation of 7-Deaza Purine Modified Oligonucleotides Containing a Repeating Motif. Int. J. Mass. Spectrom. 2002, 219, 139-150.

51. Dunbar, R. C. Kinetics of Low-Intensity Infrared Laser Photodissociation. The Thermal Model and Application of the Tolman Theorem. J. Chem. Phys. 1991, 95, 2537.

52. Affolter, M.; Watts, J. D.; Krebs, D. L.; Aebersold, R. Evaluation of Two-Dimensional Phosphopeptide Maps by Electrospray Ionization Mass Spectrometry of Recovered Peptides. Anal. Biochem. 1994, 223, 74-81. 